\title{
O TRATAMENTO DE SINTOMAS DE ANSIEDADE BASEADO NA MÚSICA E TERAPIA COGNITIVO-COMPORTAMENTAL
}

\section{Treatment of music-based anxiety symptoms and Cognitive Behavioral Therapy}

\author{
Wildson Cardoso Assunção - Universidade de Gurupi/Brasil \\ Daniel Carlos Carvalho de Castro - Associação de Pais e Amigos dos Excepcionais de \\ Porto Nacional/TO/Brasil \\ Kariely Vegin Reis - Hospital Maternidade Dona Regina de Palmas/TO/Brasil \\ Weverson Cardoso de Jesus- Universidade Federal do Tocantins/TO/Brasil
}

RESUMO: A música promove efeitos das mais diversas classes na mente humana. É praticamente impossível falar sobre fatores que influenciam o comportamento humano sem que haja, em algum momento, algo relacionado à música. Pesquisas tem sugerido que a música pode ser um aliado em tratamento de sintomas de ansiedade através da Terapia Cognitivo-Comportamental - TCC. Objetivos: Descrever e discutir sobre a eficácia do uso da música em Terapia Cognitivo-Comportamental no tratamento de sintomas de ansiedade. Metodologia: O presente estudo fez uso da revisão narrativa da literatura que possibilita descrever e discutir determinado assunto de forma eficiente, fornecendo subsídios para a discussão de uma perspectiva teórica. Utilizou-se uma abordagem descritiva e qualitativa. Resultados e discussão: Boa parte das pesquisas descrevia o uso terapêutico da música no campo da musicoterapia, que é um campo autônomo e independente, com métodos de intervenção próprios. Foi necessário descrever e considerar que há uma diferença entre musicoterapia e terapias com música ou música em terapia. O uso da música pela TCC é requisitado, descrito e recomendado, apresentando bons resultados. Conclusão: A literatura apresenta o uso da música em TCC como um recurso possível e com bons resultados no tratamento de sintomas de ansiedade e outros aspectos cognitivos disfuncionais. Foi encontrada uma diferença clara entre musicoterapia e terapia com música, no entanto, não foram encontrados estudos que apresentassem essa discrepância de forma direta e sistematizada.

Palavras-chave: Terapia Cognitivo-Comportamental. Musicoterapia. Ansiedade.

ABSTRACT: Music promotes effects of the most diverse classes on the human mind. It is practically impossible to talk about factors that influence human behavior without something related to music at some point. Research has suggested that music may be an ally in treating anxiety symptoms through cognitive behavioral therapy - CBT. Objectives: Describe and discuss the effectiveness of the use of music in cognitive behavioral therapy in treating anxiety symptoms. Methodology: The present study made use of the narrative literature review that allows describing and discussing a given subject efficiently, providing subsidies for the discussion from a theoretical perspective. A descriptive and qualitative approach was used. Results and discussion: Much of the research described the therapeutic use of music in the field of music therapy, which is an autonomous and independent field with its own intervention methods. It was necessary to describe and consider that there is a difference between music therapy and music therapy or music therapy. The use of music by CBT is requested, described and recommended, with good results. Conclusion: The literature presents the use of music in CBT as a possible resource and with good results in the treatment of anxiety symptoms

Educação, Psicologia e Interfaces, Volume 4, Número 1, p. 127-143, Janeiro/Março, 2020.

ISSN: 2594-5343. DOI: 
Wildson Cardoso Assunção, Daniel Carlos Carvalho de Castro, Kariely Vegin Reis \& Weverson Cardoso de Jesus

and other dysfunctional cognitive aspects. A clear difference was found between music therapy and therapy with music; however, no studies were found that presented this discrepancy directly and systematically.

Keywords: Cognitive behavioral therapy. Music therapy. Anxiety.

\section{INTRODUÇÃO}

A música promove efeitos das mais diversas classes na mente humana. É praticamente impossível descrever fatores que influenciam no comportamento e na cultura humana sem que haja, em algum momento, algo relacionado à música.

Estudos comprovam que a música é atuante no cérebro, produzindo estímulos e efeitos diversos na cognição, linguagem, na cultura e em muitos outros aspectos (CASAROTTO; VARGAS; MELLO-CARPES, 2017). Em um passado não muito remoto a música foi utilizada como uma das diversas formas de comunicação à distância de muitas civilizações.

Ao longo dos séculos até os dias de hoje, aquelas formas de comunicação através de som se desenvolveram e desde então vem se aperfeiçoando e sendo criada, recriada, esgotada e reinventada (SCHELLENBERG; WEISS, 2013).

Históricamente após essas reinvenções a música passou a ser estruturada e foi utilizada como forma de entretenimento (MADALOZZO, 2014), tendo uma presença bastante forte em diversos seguimentos da cultur humana. Durante muitos séculos esse tipo de música denominada música erudita era voltada especialmente às altas classes da sociedade. Essa classe musical é composta por instrumentos diversos e vozes humanas.

Ao longo do tempo vários estudos demonstraram que além do entretenimento a música poderia ser um recurso favorável em aspectos mentais, tanto em pessoas saudáveis quanto em pessoas que tiveram algum dano psicológico.

Estudos de Trimble e Hesdoffer (2017) evidenciaram que a música pode ser um recurso em vários tipos de terapias, em especial as que têm enfoque cognitivo, sendo um meio alternativo de acessar e estimular aspectos dos mais variados eixos. Concordando com essa perspectiva Trimmer, Tyo e Naeem (2016) reuniram trabalhos em várias linhas teóricas demostrando eficácia ao combinar terapias cognitivas e intervenções musicais auditivas. Dentre os resultados que apresentaram progresso estava a ansiedade.

Educação, Psicologia e Interfaces, Volume 4, Número 1, p. 127-143, Janeiro/Março, 2020. ISSN: 2594-5343. DOI: 
A ansiedade é um importante mecanismo de sobrevivência em seres humanos. Trata-se de uma resposta involuntária frente à ameaça ou perigo. Uma pessoa pode desenvolver reações de ansiedade disfuncionais, que tendem a produzir pensamentos ou concepções distorcidas da realidade, resultando prejuízos significativos (WIEDEMANN, 2015).

Essas reações disfuncionais podem existir devido a experiências aversivas ou a fatores genéticos. Em todo caso, o uso de terapias com enfoque cognitivo dispõe de vários recursos que podem ajudar a reduzir danos e estabelecer aprendizagem ou reabilitação cognitiva.

De acordo com Assunção e Da Silva (2019) a Terapia Cognitivo-Comportamental - TCC possui um aparato metodológico direto e objetivo que trabalha demandas atuais que estejam causando prejuízo na vida da pessoa. Para esses autores a TCC é a abordagem mais indicada no tratamento de transtornos ou sintomas de ansiedade.

São frequentes os estudos sobre o uso da música em tratamentos psicológicos ou de outras áreas da saúde, onde o uso de música erudita e instrumental ou ambiente é mais utilizado. Dannenberg (2010) menciona que na música erudita a predominância de melodias em um formato composto apenas por instrumentos trabalhados na própria sessão de terapia cognitiva seria suficiente para facilitar o processo de modificação de pensamento e redução da ansiedade.

Pessoas que ouvem música ambiente ou erudita instrumental durante alguma terapia têm sua ansiedade reduzida (FIRMEZA et al. 2016). Isso pode favorecer técnicas de intervenções como a geração de imagens mentais, devido à necessidade de concentração e de treinamento psicológico a qual o paciente é submetido (WRIGHT; BASCO; THASE, 2008).

Não há restrição quanto aos gêneros musicais que podem ser usados em procedimentos terapêuticos, mas pesquisadores concordam sobre o uso de música com som ambiente (sons da natureza) ou música erudita instrumental. Esses estilos aumentam níveis de concentração, uma vez que a ausência de vozes humanas supostamente não influenciaria (YEOH, 2016) ou evocaria lembranças ou pensamentos, quesitos estes que ficam comprometidos em pessoas com sintomas de ansiedade.

Na música erudita e instrumental o compositor Wolfgang Amadeus Mozart (17561791) é reconhecido como um dos maiores nomes da história por sua variedade musical 
Wildson Cardoso Assunção, Daniel Carlos Carvalho de Castro, Kariely Vegin Reis \& Weverson Cardoso de Jesus

e pelas mais de 600 obras compostas ao longo de sua vida (ASHOORI; JANKOVIC, 2007).

Schellenberg e Weiss (2013) apresentaram estudos que mostraram a eficácia das composições de Mozart no ambiente terapêutico, sendo eficaz em técnicas de relaxamento, em tomadas de decisões, em exercícios de imagens mentais, em manejo de ansiedade e muitas outras questões associadas.

Sintomas disfuncionais de ansiedade ocorrem devido a diversas causas, levando a pessoa a manifestar sentimentos e comportamentos deficitários diversos, podendo comprometer o desempenho psicológico, físico e aspectos da linguagem.

Diante desses dados foram levantadas as seguintes questões: "Há possibilidades do uso da música em intervenções da TCC em tratamentos de sintomas de ansiedade?” e “O que a literatura diz a respeito do uso da música TCC?”. Baseando-se nesses questionamentos, esse estudo teve como objetivo descrever e discutir sobre a eficácia do uso da música em Terapia Cognitivo-Comportamental, como recurso em tratamento de sintomas de ansiedade.

\section{METODOLOGIA}

O presente estudo fez uso da revisão narrativa da literatura. De acordo com Assunção e Da Silva (2019) pode descrever e discutir determinado assunto de forma eficiente, fornecendo subsídios para a discussão de uma perspectiva teórica sem estabelecer critérios replicáveis ou esgotamento das buscas nas bases de dados.

Utilizou-se uma abordagem descritiva e qualitativa. Foi dada ênfase em publicações científicas disponibilizadas em base de dados internacionais como Google Acadêmico, Scielo, periódicos, sites de universidades, repositórios, revistas científicas e no portal researchgate. O levantamento do material bibliográfico foi realizado de acordo com os critérios de inclusão e exclusão.

Foram selecionados os trabalhos mais recentes possíveis, no entanto, não foi delimitado um critério para o período da publicação das pesquisas, tendo em vista que o presente estudo teve a proposta de descrever e resgatar assuntos específicos que poderiam ser históricos.

Os critérios de seleção de inclusão adotados foram: Publicações completas; públicos; nos idiomas: Português, Inglês e Alemão. Utilizando os seguintes descritores,

Educação, Psicologia e Interfaces, Volume 4, Número 1, p. 127-143, Janeiro/Março, 2020. ISSN: 2594-5343. DOI: 
em Português, Inglês e Alemão e suas combinações: Terapia Cognitivo-Comportamental; Musicoterapia; Ansiedade.

Os critérios de exclusão adotados foram: Publicações e/ou publicações pagas; Publicações que não se relacionavam ao escopo desse estudo; Publicações com abordagens quantitativas; Resumos; Publicações incompletas.

Após a seleção de publicações houve novamente a leitura dos resumos das pesquisas com intuito de selecionar apenas as publicações que fariam parte do corpo do presente estudo, dessa forma, foram excluídas as publicações duplicadas e em mais de uma base de dados ou que conteúdo, análises, conceitos ou objetivos semelhantes.

\section{TCC DA ANSIEDADE}

A ansiedade pode ser compreendida como uma série de alterações funcionais no comportamento que garante reações voluntárias e involuntárias e prepara o organismo para a autoproteção. É considerada uma ferramenta e mecanismo de sobrevivência, necessário para sobrevivência não somente da espécie humana, mas também a de muitos outros animais (SWIFT et al. 2014).

Anomalias funcionais e estruturais no cerebelo promovem grande parte das disfunções no comportamento (BALDAÇARA et al. 2008). Várias funções fisiológicas são ativadas em momentos de ansiedade.

Graeff e Junior (2010) discorrem sobre aspectos fisiológicos da ansiedade, focando o eixo hipotálamo-hipófise-adrenal - HPA na ansiedade generalizada e em transtorno de pânico. De acordo com esses autores os hormônios adrenocorticotrópico, cortisol e prolactina não ativam o HPA em ataques de pânico em comparação com a ansiedade generalizada.

Na perspectiva da TCC, a ansiedade é vista do ponto de vista biopsicológico. É apresentada por muitos autores como "reação de luta ou fuga" (GRAEFF, 2007), onde o organismo percebe, enfrenta ou foge do perigo eminente. A disfuncionalidade desse mecanismo ocorre devido vários fatores, envolvendo experiências subjetivas, processos neuropsicológicos e também comportamentais, este ultimo, vinculado a alterações distorcidas na cognição, os erros cognitivos (EBRAHIMI et al. 2019).

Barcellos et al. (2017, p. 3) conceituam a ansiedade em seu estado comprometido como "uma combinação variável de sintomas físicos, pensamentos catastróficos e alterações de comportamento".

Educação, Psicologia e Interfaces, Volume 4, Número 1, p. 127-143, Janeiro/Março, 2020. ISSN: 2594-5343. DOI: 
Entende-se por sintoma um tipo de sinal, que é a primeira manifestação perceptível de alguma alteração no organismo. De acordo com o dicionário de termos médicos, essa alteração indica que há alguma doença ou distúrbio. Ainda de acordo com o dicionário, distúrbio seria uma perturbação do estado natural de uma pessoa (A\&C BLACK, 2004). Dessa forma, compreendemos sintomas da ansiedade como início de algo que pode ser estabelecido em caso de não tratamento.

Essas perturbações no organismo envolvem alterações de sensações e percepção, alteração respiratória como a hiperventilação, asfixia, tontura, medo, confusão, taquicardia, náusea, sudorese, perda de apetite e perda de peso, limitações subjetivamente percebidas, distúrbios de afeto, humor e distúrbios do sono (SCHAAF, 2015).

De acordo com Ebrahimi, et al. (2019) as teorias cognitivas têm apontado muitos desses sintomas devido aos erros cognitivos. Esses erros cognitivos são vistos como desencadeadores responsáveis também pelos comportamentos deficitários e transtornos relacionados.

A ansiedade disfuncional é um problema no processamento das informações relevantes do ambiente que confundem a realidade causando processamentos desorganizados e antecipados de respostas involuntárias, que por sua vez causam medo.

Todo esse processo atinge as crenças nucleares de uma pessoa, fazendo com que haja a criação ou modificação de outras crenças. Uma vez que essas novas crenças foram baseadas em processos disfuncionais, essas crenças também permanecem disfuncionais.

Em TCC utiliza-se o termo esquemas ${ }^{1}$ para se referir às crenças nucleares de uma pessoa. Essas crenças atuam como base fundamental para produzir a maneira que a pessoa vê seu mundo interno e externo (WRIGHT; BASCO; THASE, 2008). Esquemas são grandes estruturas cognitivas que desempenham um importante papel para a vida humana, é através disso que se torna possível perceber, sentir, selecionar, filtrar, assimilar e atribuir significado às informações provenientes do ambiente.

Margraf e Schneider (2009) pedem atenção na questão dos esquemas, pois eles envolvem não somente a questão do eu com o mundo externo, mas também do eu consigo mesmo, havendo variações entre o ativar e o desativar desses esquemas de acordo com o processo terapêutico.

\footnotetext{
${ }^{1}$ Não confundir com esquemas de reforçamento.
}

Educação, Psicologia e Interfaces, Volume 4, Número 1, p. 127-143, Janeiro/Março, 2020. ISSN: 2594-5343. DOI: 
Além disso, evidências científicas mostram que a genética é um fator que precisa ser considerado na compreeção dos esquemas, pois a influencia em aspectos cognitivos e comportamentais do individuo pode provocar aumento do ácido gama-aminobutírico GABA que aumenta níveis de estresse e ansiedade (MARGIS et al. 2003).

A TCC possibilita o ensino de seus métodos aos pacientes, ensinando-os a refletir sobre o próprio pensamento para (re)educação cognitiva e controle dos pensamentos (WRIGHT; BASCO; THASE, 2008).

Ainda de acordo com esses autores o desenvolvimento de esquemas é moldado com base nas experiências coletivas ou subjetivas e interações sociais. A falta de incentivo e motivação para certas ocasiões podem comprometer os esquemas de uma pessoa. Compreender e estudar esses fatores ajuda o terapeuta a formular hipóteses e planos de tratamento e educação ao paciente.

Vinculados aos esquemas, os pensamentos automáticos são os antecessores de comportamentos deficitários. Huffziger et al. (2008) afirmam que pensamentos automáticos representam causa maior de transtornos depressivos. Apesar de que a ansiedade é tida como um polo oposto à depressão, seus sintomas estão presentes em boa parte de transtornos depressivos (BRASIL; PONDÉ, 2009, ASSUNÇÃO; DA SILVA, 2019).

A literatura mostra que a TCC é a abordagem da psicologia mais indicada para intervir em situações como distúrbios de comportamento e de ansiedade. No Brasil a demanda aumentou significativamente nas ultimas décadas em consultórios médicos e de psicologia (ROEMER; ORSILLO, 2010).

\section{USO DA MÚSICA EM TCC}

Estudos citados por Sampaio, Loureiro e Gomes (2015) definem um campo científico independente denominado de musicoterapia como o uso profissional da música como forma de intervenção que proporciona, dentre diversos aspectos, qualidade de vida e de saúde.

Não se sabe exatamente como ou onde a música como forma de tratamento começou a ser aplicada. Trata-se de um aspecto tão antigo quanto à própria criação da música. As variações sobre o conceito variam e variaram bastante. Dobrzyńska et al. (2006) afirmam que uma definição científica para a musicoterapia surgiu logo após a Segunda Guerra Mundial, por volta de 1950. De acordo com esses autores a musicoterapia

Educação, Psicologia e Interfaces, Volume 4, Número 1, p. 127-143, Janeiro/Março, 2020. ISSN: 2594-5343. DOI: 
Wildson Cardoso Assunção, Daniel Carlos Carvalho de Castro, Kariely Vegin Reis \& Weverson Cardoso de Jesus

é bastante usada para promoção, adaptação e reabilitação de aspectos relacionados à saúde mental.

Estudos de Tüpker (2001) colocam que o desenvolvimento da musicoterapia baseiou-se em uma morfologia e uma junção de saberes de diversas áreas do conhecimento, historicamente.

A musicoterapia pode ser aplicada em diversos contextos, como em grupo, em tratamentos individuais, em ambientes clínicos e hospitalares, ou mesmo como parte de uma (re)educação ao paciente. No Brasil existe uma formação em nível de especialização, multidisciplinar e específica para atuação do musicoterapeuta.

No campo da psicologia, Trimmer, Naeem e Tyo (2016) evidenciam que o uso terapêutico da música em TCC é mostrado promissor tanto na prática quanto em pesquisas, especialmente no tratamento de depressão e ansiedade.

Esses autores realizaram um estudo em um grupo de autoajuda por nove semanas com pessoas que apresentavam sintomas leves ou moderados de ansiedade e/ou depressão. Esse estudo demonstrou eficiência nos resultados.

Essas terapias podem aumentar o engajamento, a adesão e o baixo custo, além de atender aos clientes em seus níveis cognitivos e emocionais [...]. A música pode ser pensada como uma porta de entrada para a psico-educação e autoajuda, ampliando a ideia de entrega terapêutica [...] (TRIMMER, NAEEM; TYO, 2016, p. 4, Tradução nossa)

Hagemann, Martin e Neme (2019) demonstraram que pacientes apresentaram redução significativa dos sintomas de depressão através do uso da musica em terapia. Os tratamentos de depressão contam com diversas técnicas da TCC (ASSUNÇÃO; DA SILVA, 2019), no entanto, contextos específicos podem demandar novos aspectos no tratamento com intuito de não cansar o paciente a exercícios que podem ser interpretados como repetitivos. Dentre as possíveis intervenções em depressão e ansiedade Burin e Osório (2017) orientam estratégias de enfrentamento como técnicas de respiração e técnicas de relaxamento.

Cully e Teten (2008) em meio a esse tratamento, uma boa estratégia é orientar o cantar e ouvir música, por exemplo. Aalbers et al. (2017) aprofundam mais as possibilidades quando orientam performances, composições ao vivo ou gravadas, e etc.

Educação, Psicologia e Interfaces, Volume 4, Número 1, p. 127-143, Janeiro/Março, 2020. ISSN: 2594-5343. DOI: 
Em seu estudo, os autores constataram melhoras significativas em quadros cognitivos disfuncionais de mais de 400 pacientes, em um curto período de tempo.

As evidências dos efeitos positivos do uso da música no contexto de intervenções em TCC são favoráveis na literatura e apresentam-se como um bom recurso terapeutico. Percebe-se que as intervenções envolvendo músicas são amplas, indo além das metodologias utilizadas pela TCC, sendo usada em diversos contextos. Isso pode favorecer ainda mais seu uso para a aceitação do paciente, especialmente pacientes que estão em contato com a psicologia pela primeira vez e ainda não tem um conceito formado.

Ainda em relação ao uso da música, cabe ressaltar uma categoria conhecida como Terapia Sonora, que é um método que proporciona estimulação do ouvido pela presença de sons constantes que tem o objetivo de reduzir hipersensibilidade em ambientes silenciosos ou zumbidos (SUZUKI et al. 2016).

De acordo com esses autores a terapia sonora, através do uso de geradores de som com ou sem amplificação auditiva, proporcionam melhora no quadro de pessoas que "ouvem" zumbido, por exemplo. Esse método faz com que a pessoa se concentre menos no Zumbido.

\section{MUSICOTERAPIA E TERAPIA COM MÚSICA}

O levantamento bibliográfico foi realizado em diversos portais, dentre eles o Google Acadêmico, repositórios, revistas científicas, monografias e teses de mestrado e doutorado. As buscas ocorreram através dos descritores "Terapia CognitivoComportamental", "Musicoterapia", "Ansiedade" e das combinações desses termos com outras palavras chaves que indicassem o caminho metodológico para recuperar arquivos que tratassem das perspectivas dos objetivos.

Após a busca pelas pesquisas, foram selecionadas 40 publicações que compuseram o corpo do trabalho. Através do levantamento bibliográfico foram encontrados dados satisfatórios que relacionavam TCC e a música como um recurso.

No entanto, boa parte dessas pesquisas descrevia o uso da música no campo da musicoterapia que de acordo com Carreras (2015) é um campo independente com suas próprias abordagens, metodologias e técnicas.

Educação, Psicologia e Interfaces, Volume 4, Número 1, p. 127-143, Janeiro/Março, 2020. ISSN: 2594-5343. DOI: 
Dessa forma, para fins didáticos, foi preciso considerar que há uma diferença entre a aplicabilidade de musicoterapia (como um campo distinto) e terapia com música ou música em terapia (onde a música seria recurso terapeutico).

A terminologia em inglês "Music Therapy" pode se referir a ambas, mas ao considerar essa discrepância é possível gerar outras possibilidades de uso de música em ambiente terapeutico. A questão da tradução literal dessa terminologia pode ser entendida como um aspecto semântico da linguagem, mas na língua Portuguesa, é possível criar um paralelo e estabelecer uma diferença de conceitos (Tabela 1).

Tabela 1. Comparativo entre Musicoterapia e Terapia com Música

\begin{tabular}{|c|c|}
\hline MUSICOTERAPIA & TERAPIA COM MÚSICA \\
\hline $\begin{array}{l}\text { Pode ser usada por diversas áreas da saúde, } \\
\text { dentre elas: Psicologia, Fisioterapia, } \\
\text { Fonoaudiologia, etc. }\end{array}$ & $\begin{array}{l}\text { Terapia que faz uso de música, não } \\
\text { necessariamente como em } \\
\text { musicoterapia. }\end{array}$ \\
\hline $\begin{array}{l}\text { Estimula, através do som e da produção de } \\
\text { som, canais de relação entre o homem e o } \\
\text { meio externo, minimizando aspectos motores } \\
\text { e mentais deficitários. }\end{array}$ & \multirow{2}{*}{$\begin{array}{l}\text { Terapia sorona que evoca lembranças } \\
\text { e pensamentos diversos, que } \\
\text { possibilita espaço favorável para } \\
\text { intervenções em sintomas de } \\
\text { ansiedade, depressão. }\end{array}$} \\
\hline \multirow{2}{*}{$\begin{array}{l}\text { Área autônoma com seus próprios métodos e } \\
\text { técnicas. }\end{array}$} & \\
\hline & \multirow{2}{*}{$\begin{array}{l}\text { Seu uso depende da abordagem, dos } \\
\text { métodos do profissional e dos } \\
\text { objetivos de tratamento. }\end{array}$} \\
\hline \multirow{2}{*}{$\begin{array}{l}\text { Envolve processos comunicativos, expressão } \\
\text { corporal, melodia, ritimo, harmonia } \\
\text { possibilitando evolução ou reabilitação em } \\
\text { aspectos motores e mentais. }\end{array}$} & \\
\hline & \multirow{2}{*}{$\begin{array}{l}\text { Pode ser limitada apenas ao uso de } \\
\text { música fundo no ambiente, ou } \\
\text { complementando em alguma técnica } \\
\text { ou procedimento. }\end{array}$} \\
\hline $\begin{array}{l}\text { Envolve o uso de recursos como } \\
\text { instrumentos, criação de instrumentos, leitura } \\
\text { musical e aspectos de ensino/aprendizagem. }\end{array}$ & \\
\hline Eficaz em saúde mental. & Eficaz em saúde mental. \\
\hline Requer de título de especialização. & $\begin{array}{l}\text { Não requer de título específico de } \\
\text { especialização. }\end{array}$ \\
\hline $\begin{array}{l}\text { Possibilidades de uso em terapias grupais, } \\
\text { onde são trabalhadas atividades motoras que } \\
\text { desenvolvem funções potenciais do } \\
\text { indivíduo para qualidade de vida. }\end{array}$ & $\begin{array}{l}\text { Há possibilidades de uso em terapias } \\
\text { grupais, no entanto, em casos de } \\
\text { transtornos de ansiedade, recomenda- } \\
\text { se o seu uso individual. }\end{array}$ \\
\hline Diversos estilos musicais. & $\begin{array}{l}\text { Recomenda-se música instrumental ou } \\
\text { ambiente. }\end{array}$ \\
\hline
\end{tabular}

Fonte: Dados da pesquisa.

As pesquisas que expõe a utilização da música no campo de áreas relacionadas à saúde mental mostram eficiência em terapias para o tratamento de transtornos por uso de

Educação, Psicologia e Interfaces, Volume 4, Número 1, p. 127-143, Janeiro/Março, 2020. ISSN: 2594-5343. DOI: 
substâncias (HOHMANN et al. 2017) autismo (SAMPAIO; LOUREIRO; GOMES, 2015), esquizofrenia (GERETSEGGER et al. 2017) depressão (ANJOS et al. 2017) e ansiedade, aspectos estes que são frequentemente estudados e trabalhados em TCC.

Consideramos como tratamento de sintomas da ansiedade mencionado nesse estudo apenas voltado ao sintoma, compreendendo por sintoma como sinais de alterações no funcionamento do corpo (A\&C BLACK, 2004). Entendendo que sintomas são etapas iniciais, que devem ser compreendidos e estudados antes de qualquer diagnóstico ou plano de terapêutico.

O uso de musicoterapia é realizado por profissionais de diversas áreas, o que torna sua atuação ampla. O campo da psicologia utiliza como recursos em tratamentos específicos e não necessariamente da mesma forma que um musicoterapeuta faria.

A tabela 1 apresentou um comparativo de como foi identificada e realizada a discrepância entre musicoterapia e terapia com música, em uma perspectiva generalizada, de atuação e com base na reflexão teórica das pesquisas levantadas.

Como visto na tabela acima, o tipo e objetivo de aplicabilidade da musicoterapia e terapia com música se diferenciam em vários de seus aspectos, aplicabilidades e metodologias, é preciso considerar também que nem todos os terapeutas que utilizam a música em terapia são musicoterapeutas.

Em relação ao tratamento de sintomas da ansiedade, percebe-se uso de música como recurso esconjuntado ou agregado às técnicas de uso comum da TCC. O tratamento dos sintomas da ansiedade não necessariamente é como o tratamento de transtornos da ansiedade, mas se não forem tratados, podem evoluir de forma comprometedora (BYSTRITSKY et al. 2013).

\section{CONSIDERAÇÕES FINAIS}

A literatura apresenta o uso da música em TCC como um recurso possível e com bons resultados no tratamento de sintomas de ansiedade e outros aspectos cognitivos disfuncionais. Foi encontrada uma diferença clara entre musicoterapia e terapia com música, no entanto, não foram encontradas publicações que apresentassem essa discrepância de forma direta e sistematizada.

A hipótese inicial do presente estudo considerava descrever apenas sobre possibilidades do uso da música em intervenções da TCC em tratamentos de sintomas de ansiedade, a literatura mostrou eficiência, além de uma ampla aplicabilidade, no entanto,

Educação, Psicologia e Interfaces, Volume 4, Número 1, p. 127-143, Janeiro/Março, 2020. ISSN: 2594-5343. DOI: 
Wildson Cardoso Assunção, Daniel Carlos Carvalho de Castro, Kariely Vegin Reis \& Weverson Cardoso de Jesus

essas mesmas possibilidades nos levou à necessidade de realizar uma contextualização entre musicoterapia e o uso da música em intervenções da psicologia. Sugerimos mais pesquisas empíricas e teóricas sobre esses dados para avaliar situações e necessidades específicas.

O perfil das publicações analisadas evidencia uma discussão qualitativa satisfatória em alguns aspectos. Por outro lado, apresentam déficit em se tratando de descrição de procedimentos metodológicos em relação ao tratamento, nos levando a refletir sobre "como os terapeutas que fazem uso da TCC poderiam elaborar um plano de intervenção subsidiado, utilizando a música sem uma referência sólida?” esse quesito precisa ser analisado e desenvolvido com mais precisão.

O presente estudo fez apenas um pequeno recorte na literatura, recomenda-se que haja mais discussões sobre uso da música em TCC e alternativas de tratamentos em pacientes que apresentam sintomas, distúrbios ou transtornos de ansiedade, meios alternativos, para populações específicas, que visem contribuir para sua qualidade de vida e saúde mental.

\section{REFERÊNCIAS}

AALBERS, S. et al. Music therapy for depression. Cochrane Database of Systematic Reviews, 2017. Disponível em:

<https://www.cochranelibrary.com/cdsr/doi/10.1002/14651858.CD004517.pub3/abstrac t>. Acesso em 06/06/2019.

A\&C BLACK. Dictionary of Medical Terms. 4th edition. 2004. A \& C Black. Disponível em: <alexabe.pbworks.com/f/Dictionary+of+Medical+Terms+4th+Ed.+(Malestrom).pdf>. Acesso em: 06/06/2019.

ANJOS, A. G.; et al . Musicoterapia como estratégia de intervenção psicológica com crianças: uma revisão da literatura. Rev. Interinst. Psicol. v. 10, n. 2, p. 228-238. 2017. Disponível em <http://pepsic.bvsalud.org/pdf/gerais/v10n2/08.pdf >. Acesso em: 09/09/2019.

ASHOORI, A.; JANKOVIC, J. Mozart's movements and behaviour: A case of Tourette's syndrome?. 2007. Journal of neurology, neurosurgery, and psychiatry. 78(11):1171-5. Disponível em:

<https://www.researchgate.net/publication/5905691_Mozart's_movements_and_behavi our_A_case_of_Tourette's_syndrome>. Acesso em: 15/05/2019.

ASSUNÇÃO, W. C.; DA SILVA, J. B. F. Aplicabilidade das técnicas da terapia cognitivocomportamental no tratamento de depressão e ansiedade. Revista Educação, Psicologia e Interfaces, v. 3, n.1, p. 77-94, 2019. Disponível em:

Educação, Psicologia e Interfaces, Volume 4, Número 1, p. 127-143, Janeiro/Março, 2020. ISSN: 2594-5343. DOI: 
<http://educacaoepsicologia.emnuvens.com.br/edupsi/article/view/113/91>. Acesso em: 15/09/2019.

BALDAÇARA, L. et al. Cerebellum and psychiatric disorders. Rev. Bras. Psiquiatr. vol.30 no.3. pp, 281-9. 2008. Disponível em:

<www.scielo.br/pdf/rbp/v30n3/a16v30n3.pdf>. Acesso em: 17/05/2019.

BARCELLOS, M. T. et al. Telecondutas - transtornos de ansiedade, transtorno de estresse pós-traumático e transtorno obsessivo-compulsivo. 2017.

TelessaúdeRS/UFRGS. Disponível em:

<https://www.ufrgs.br/telessauders/documentos/telecondutas/Telecondutas_Ansiedade_ 20170331.pdf >. Acesso em: 17/05/2019.

BRASIL, I. S. P. S.; PONDÉ, M. P. Sintomas ansiosos e depressivos e sua correlação com intensidade da dor em pacientes com neuropatia periférica. Rev Psiquiatr RS. 2009;31(1):24-31. Disponível em: <www.scielo.br/pdf/rprs/v31n1/v31n1a07.pdf>. Acesso em: 18/07/2019.

BURIN, A. B.; OSÓRIO, F. L. Music performance anxiety: a critical review of etiological aspects, perceived causes, coping strategies and treatment. Arch Clin Psychiatry. 2017;44(5):127-33. Disponível em: <www.scielo.br/pdf/rpc/v44n5/01016083-rpc-44-5-0127.pdf>. Acesso em 14/07/2019.

BYSTRITSKY, A. et al. Current Diagnosis and Treatment of Anxiety Disorders. P T. 2013 Jan; 38(1): 30-38, 41-44, 57. Disponível em:

<https://www.ncbi.nlm.nih.gov/pmc/articles/PMC3628173/>. Acesso em: 13/05/2019.

CASAROTTO, F. D. et al. Música e seus efeitos sobre o cérebro: uma abordagem da neurociência junto a escolares. 2017. Revista ELO - Diálogos em Extensão, Volume 06, número 02. Disponível em: <https://doi.org/10.21284/elo.v6i2.243>. Acesso em: 05/03/2019.

BRASIL. Resolução n ${ }^{\circ} 466$, de 12 de dezembro de 2012. Conselho Nacional de Saúde CNS. Disponível em: <conselho.saude.gov.br/resolucoes/2012/Reso466.pdf>. Acesso em: 13/03/2019.

CULLY, J. A.; TETEN, A. L. A therapist's guide to brief cognitive behavioral therapy. Department of Veterans Affairs, South Central Mental Illness Research, Education, and Clinical Center (MIRECC), 2008. Disponível em:

<https://www.mirecc.va.gov/visn16/docs/therapists_guide_to_brief_cbtmanual.pdf>. Acesso em: 14/07/2019.

DANNENBERG, R. B. Style in Music. Preprint from The Structure of Style:

Algorithmic Approaches to Understanding Manner and Meaning, Berlin: SpringerVerlag, 2010, pp. 45-58. Disponível em: <https://www.cs.cmu.edu/ rbd/papers/rbdstyle-2009.pdf >. Acesso em: 15/08/2019.

DOBRZYŃSKA, E.; et al. Music Therapy - History, definitions and application. Archives of Psychiatry and Psychotherapy 8(1):47-52. 2006. Disponível em:

Educação, Psicologia e Interfaces, Volume 4, Número 1, p. 127-143, Janeiro/Março, 2020. ISSN: 2594-5343. DOI: 
Wildson Cardoso Assunção, Daniel Carlos Carvalho de Castro, Kariely Vegin Reis \& Weverson Cardoso de Jesus

<https://www.researchgate.net/publication/282647747_Music_Therapy_-

_History_definitions_and_application>. Acesso em: 15/08/2019.

EBRAHIMI, L. S. Cognitive Errors and Psychological Resilience in Patients With Social Anxiety and Obsessive-Compulsive Disorder: A Cross-Sectional Study. Hosp

Pract Res. 2019 Feb;4(1):25-30. Disponível em:

<www.jhpr.ir/article_82174_58bfff4a56fdaaab9acf0fd03b7100f6.pdf>. Acesso em: 17/05/2019.

FIRMEZA, M. A. et al. Uso da música no controle da ansiedade em ambulatório de cabeça e pescoço: Ensaio clínico randomizado. Rev. Esc. Enferm. USP, 2017, pp 1-8. Disponível em: <www.scielo.br/pdf/reeusp/v51/pt_1980-220X-reeusp-51-e03201.pdf>. Acesso em: 14/02/2019.

GERETSEGGER, M. et al. Music therapy for people with schizophrenia and schizophrenia-like disorders. Cochrane Systematic Review. Disponível em: <https://www.cochranelibrary.com/cdsr/doi/10.1002/14651858.CD004025.pub4/full>. Acesso em 14/07/2019.

GRAEFF, F. G. Anxiety, panic and the hypothalamic-pituitary-adrenal axis. Rev. Bras. Psiquiatr. vol.29 suppl.1. pp. S3-S6 2007. Disponível em:

<http://www.scielo.br/pdf/rbp/v29s1/en_a02v20s1.pdf>. Acesso em: 17/05/2019.

GRAEFF, F. G.; JUNIOR, H. Z. The hypothalamic-pituitary-adrenal axis in anxiety and panic. Psychology \& Neuroscience, 2010, 3, 1, 3 - 8. Disponível em:

<www.scielo.br/pdf/pn/v3n1/v3n1a02>. Acesso em: 17/05/2019.

HAGEMANN, P. M. S.; MARTIN, L. M.; NEME, C. M. B. O efeito da musicoterapia na qualidade de vida e nos sintomas de depressão de pacientes em hemodiálise. Braz. J. Nephrol. (J. Bras. Nefrol.) 2019;41(1):74-82. Disponível em: <www.scielo.br/pdf/jbn/v41n1/pt_2175-8239-jbn-2018-0023.pdf>. Acesso em 14/07/2019.

HOHMANN, L. et al. Effects of music therapy and music-based interventions in the treatment of substance use disorders: A systematic review. PLoS ONE, 2017. 12(11). Disponível em:

<https://journals.plos.org/plosone/article/file?id=10.1371/journal.pone.0187363\&type= printable>. Acesso em 15/10/2019.

HUFFZIGER, et al. Evaluation eines deutschsprachigen Instrumentes zur Erfassung positiver und negativer automatischer Gedanken bei Kindern und Jugendlichen.

Zeitschrift für Klinische Psychologie und Psychotherapie. 37(4):255-262, 2008.

Disponível em:

<https://www.researchgate.net/publication/247398651_Evaluation_eines_deutschsprach igen_Instrumentes_zur_Erfassung_positiver_und_negativer_automatischer_Gedanken_ bei_Kindern_und_Jugendlichenf>. Acesso em: 18/07/2019.

Educação, Psicologia e Interfaces, Volume 4, Número 1, p. 127-143, Janeiro/Março, 2020.

ISSN: 2594-5343. DOI: 
MADALOZZO, T. Música do Século XX. 2014. Unicentro. Disponível em: <repositorio.unicentro.br:8080/jspui/bitstream/123456789/949/5/Música\%20no\%20séc ulo\%20XX.pdf>. Acesso em: 12/03/2019.

MARGIS, R. et al. Relação entre estressores, estresse e ansiedade. R. Psiquiatr RS, 25'(suplemento 1): 65-74, abril 2003. Disponível em:

<www.scielo.br/pdf/rprs/v25s1/a08v25s1>. Acesso em: 18/07/2019.

MARGRAF, J.; SCHNEIDER, S. Kognitionsdiagnostik. Lehrbuch der

Verhaltenstherapie, Band 1. 3. Auflage. 2009. Disponível em:

<https://www.kli.psy.ruhr-uni-bochum.de/klipsy/public/margraf\%20books/Band1Kapitel23.pdf>. Acesso em: 18/07/2019.

ROEMER, L.; ORSILLO, S. M. A prática da TCC baseada em mindfulness e aceitação. Artmed, 2010.

SAMPAIO, R. T.; LOUREIRO, C. M. V.; GOMES, C. M. A. A Musicoterapia e o Transtorno do Espectro do Autismo: uma abordagem informada pelas neurociências para a prática clínica. Per Musi. Belo Horizonte, n.32, 2015, p.137-170. Disponível em: <www.scielo.br/pdf/pm/n32/1517-7599-pm-32-0137.pdf>. Acesso em: 18/07/2019.

SCHAAF, V. H. Angst-Schwindel und Schwindel-Angst. Drhschaaf. daz Nr. 71, III/2015. Disponível em: <www.drhschaaf.de/Schaaf\%20Angst-

Schwinde1\%20und\%20Schwindel-Angst\%20daz\%2071_S4-10.pdf>. Acesso em: 17/05/2019.

SCHELLENBERG, E. G.; WEISS, M. W. Music and Cognitive Abilities. 2013.

Elsevier Inc. Disponível em:

$<$ https://www.utm.utoronto.ca/ w3psygs/SchellenbergWeissPoM.pdf >. Acesso em: $15 / 03 / 2019$.

SCHELLENBERG, E. G.; WEISS, M. W. Music and cognitive abilities. 2013. The psychology of music, pp. 499-550. Disponível em:

<https://psycnet.apa.org/record/2012-14631-012>. Acesso em: 15/05/2019.

SUZUKI, F. A. B; et al. Effectiveness of sound therapy in patients with tinnitus resistant to previous treatments: importance of adjustments. Braz J Otorhinolaryngol. 2016;82(3):297-303. Disponível em: <www.scielo.br/pdf/bjorl/v82n3/1808-8686-bjorl82-03-00297.pdf>. Acesso em 17/06/2019.

SWIFT, P. et al. Living with anxiety: Understaning the role and impact of anxiety in our lives. 2014. Mental Health Foundation. Disponível em:

<https://www.mentalhealth.org.nz/assets/A-Z/Downloads/Living-with-anxiety-reportMHF-UK-2014.pdf>. Acesso em: 17/05/2019.

TRIMBLE, M.; HESDORFFER, D. Music and the brain: the neuroscience of music and musical appreciation. Bjpsych International volume 14 number 2, 2017, pp.28-31.

Disponível em:

<https://www.researchgate.net/publication/320812687_Music_and_the_brain_the_neur oscience_of_music_and_musical_appreciation>. Acesso em: 02/05/2019. 
Wildson Cardoso Assunção, Daniel Carlos Carvalho de Castro, Kariely Vegin Reis \& Weverson Cardoso de Jesus

TRIMMER, C. G.; TYO, R.; NAEEM, F. Cognitive Behavioural Therapy-Based Music (CBT-Music) Group for Symptoms of Anxiety and Depression. Canadian journal of community mental health $=$ Revue canadienne de santé mentale communautaire 35(2):1-5. 2016. Disponível em:

<https://www.researchgate.net/publication/303395065_Cognitive_Behavioural_Therap y-Based_Music_CBT-Music_Group_for_Symptoms_of_Anxiety_and_Depression>. Acesso em 14/07/2019.

TRIMMER, C.; TYO, R.; NAEEM, F. Cognitive Behavioural Therapy-Based Music (CBT-Music) Group for Symptoms of Anxiety and Depression. Canadian Journal Of Community Mental Health, Vol. 35, N. 2, 2016. Disponível em:

<https://www.cjcmh.com/doi/abs/10.7870/cjcmh-2016-029>. Acesso em: 04/04/2019.

TÜPKER, R. Morphologisch orientierte Musiktherapie. Erschienen in: H. H. Decker-Voigt (Hrsg): Schulen der Musiktherapie, Ernst Reinhardt Verlag, München Basel 2001, S. 53-77. Disponível em: <https://www.unimuenster.de/imperia/md/content/musikpaedagogik/musiktherapie/pdfdateien/morphologisch_orientierte_musiktherapie_korr_kranz2012.pdf $>$. Acesso em: $15 / 08 / 2019$.

WIEDEMANN, K. Anxiety and Anxiety Disorders. Elsevier Ltd. Pp. 804-10 2015. Disponível em: <http://www.imhlk.com/wp-content/uploads/2018/03/Anxiety-andAnxiety-Disorders-1.pdf >. Acesso em: 15/06/2019.

WRIGHT, J. H.; BASCO, M. R.; THASE, M. E. Princípios básicos da terapia cognitivo-comportamental. Porto Alegre, RS Artmed, 2008.

YEOH, M. Effects of classical music and preferred music on mental health: an interview with Dr. Miranda Yeoh. 2016. Electronic Physician. Disponível em: <https://www.researchgate.net/publication/303013700_Effects_of_classical_music_and _preferred_music_on_mental_health_an_interview_with_Dr_Miranda_Yeoh>. Acesso em: 15/06/2019.

\section{Credenciais da/os autora/es}

ASSUNÇÃO, Wildson Cardoso. Especialista em Terapia Cognitivo-Comportamental pela Faculdade de Ciências Sociais Aplicadas de Marabá - FACIMAB, Especialista em Psicologia Organizacional e do Trabalho pela FACIMAB e Graduado em Psicologia pela Universidade de Gurupi/Brasil. E-mail: xzzyn@ @utlook.com

CASTRO, Daniel Carlos Carvalho de. Associação de Pais e Amigos dos Excepcionais de Porto Nacional/TO E-mail: fisiodanielcarlos@gmail.com

REIS, Kariely Vegin. Hospital Maternidade Dona Regina de Palmas/TO/Brasil. E-mail: karis_reis@hotmail.com

Educação, Psicologia e Interfaces, Volume 4, Número 1, p. 127-143, Janeiro/Março, 2020. ISSN: 2594-5343. DOI: 
JESUS, Weverson Cardoso de. Mestre em História pela Universidade Federal de Goiás, Graduado em História pela Universidade Federal do Tocantins, Pós-graduado em História e Cultura Afrobrasileira - Universidade Candido Mendes. Professor substituto na Universidade Federal do Tocantins/Brasil. E-mail: weversonsem@gmail.com

Endereço para correspondência: Wildson Cardoso Assunção. E-mail: xzzyn@outlook.com

Como citar este artigo (Formato ABNT): ASSUNÇÃO, Wildson Cardoso et al.. O tratamento de sintomas de ansiedade baseado na música e Terapia CognitivoComportamental. Educação, Psicologia e Interfaces, v. 4, n. 1, p. 127-143, 2020.

Recebido: 29/10/2019.

Aceito: 26/11/2019. 